\begin{tabular}{|c|l|}
\hline Title & Photoinduced phase separation with local structural ordering in organic molecular conductors \\
\hline Author(s) & Tsuchiya, S.; Nakagawa, K.; Y amada, J.; Taniguchi, H.; Toda, Y. \\
\hline Citation & $\begin{array}{l}\text { Physical Review B, 96(13), 134311 } \\
\text { https://doi.org/10.1103/PhysRevB.96.134311 }\end{array}$ \\
\hline Issue Date & 2017-10-27 \\
\hline Doc URL & http://hdl.handle.net/2115/67752 \\
\hline Rights & @2017 A merican Physical Society \\
\hline Type & article \\
\hline File Information & PhysRevB.96.134311.pdf \\
\hline
\end{tabular}

Instructions for use 


\title{
Photoinduced phase separation with local structural ordering in organic molecular conductors
}

\author{
S. Tsuchiya,,${ }^{1, *}$ K. Nakagawa, ${ }^{1}$ J. Yamada, ${ }^{2}$ H. Taniguchi, ${ }^{3}$ and Y. Toda ${ }^{1}$ \\ ${ }^{1}$ Department of Applied Physics, Hokkaido University, Sapporo, Hokkaido 060-8628, Japan \\ ${ }^{2}$ Graduate School of Material Science, University of Hyogo, Hyogo 678-1205, Japan \\ ${ }^{3}$ Department of Physics, Faculty of Science, Saitama University, Saitama 338-8570, Japan
}

(Received 23 January 2017; revised manuscript received 15 September 2017; published 27 October 2017)

\begin{abstract}
In this work, polarized pump-probe spectroscopy was carried out to investigate the effects of a structural ordering of molecules on photoinduced phase separation (PIPS) in the organic conductors $\kappa$-(BEDT-TTF) ${ }_{2} X$ $\left[X=\mathrm{Cu}\left[\mathrm{N}(\mathrm{CN})_{2}\right] \mathrm{Br}(\kappa-\mathrm{Br})\right.$ and $\left.\mathrm{Cu}(\mathrm{NCS})_{2}(\kappa-\mathrm{NCS})\right]$. We found that the anisotropic response for the probe polarization appeared at around $T_{\mathrm{g}}$, where the glasslike structural transition occurs. The anisotropy can be a result of a transient destruction of the local ordering of molecules, indicating a connection between the glasslike transition and PIPS. Moreover, we found that the PIPS response gradually develops with decreasing temperature in $\kappa$-Br, whereas it steeply increases in $\kappa$-NCS. This qualitative difference suggests that the structural ordering caused by a PIPS is more crucial in $\kappa$-NCS than in $\kappa$ - $\mathrm{Br}$.
\end{abstract}

DOI: 10.1103/PhysRevB.96.134311

\section{INTRODUCTION}

Organic molecular crystals have attracted much attention for fundamental research in strongly correlated electron systems and device applications because of their rich variety of electronic states under equilibrium [1] as well as nonequilibrium states, which can be realized via photoinduced phase transition (PIPT) [2]. Because, in organic systems, inherent molecular degrees of freedom often have a crucial effect on their electronic states [3], it is important to investigate the relationship between such degrees of freedom and the electronic system to explore different properties and functionalities.

The series of $\kappa$-(BEDT-TTF $)_{2} X$ ( $X$ : inorganic anion molecules) has been one of the most studied organic compounds because of its exotic electronic properties [4], and is similar to the cuprate superconductors [5]. In this system, several measurements indicate that a glasslike structural transition, which is associated with a ordering of BEDT-TTF or anion molecules, occurs and has a significant effect on their electronic states under equilibrium [6-10]. However, effects of the structural transition on PIPT phenomena remain unclear.

Pump-probe spectroscopy with different probe polarizations can be a suitable measurement for such nonequilibrium investigations. In the measurements, when electronic or structural ordering is present, it is destroyed transiently by pump pulse irradiation. As a result, the photoinduced carrier relaxation with recovery of the ordering is observed as enhancement of anisotropy for the probe polarization [11-13]. Thus, the measurements enable us to investigate PIPT phenomena with the inherent molecular ordering.

In this paper, we report the effect of the glasslike transition on the photoinduced phase separation (PIPS) investigated by polarized pump-probe measurements in $\kappa$-(BEDT$\mathrm{TTF}) \mathrm{Cu}\left[\mathrm{N}(\mathrm{CN})_{2}\right] \mathrm{Br}(\kappa-\mathrm{Br})$ and $\kappa-(\mathrm{BEDT}-\mathrm{TTF})_{2} \mathrm{Cu}(\mathrm{NCS})_{2}$ $(\kappa-\mathrm{NCS})$. The onset of anisotropic responses for the probe polarization is observed at $70-80 \mathrm{~K}$ in both compounds, which corresponds to the glasslike transition temperatures $T_{\mathrm{g}}$. As compared with the results from $\kappa-(\mathrm{BEDT}-\mathrm{TTF})_{2} \mathrm{Cu}\left[\mathrm{N}(\mathrm{CN})_{2}\right] \mathrm{Cl}(\kappa-\mathrm{Cl})$, the PIPS is found to

\footnotetext{
*satoshi.tsuchiya@eng.hokudai.ac.jp
}

be closely related to the structural ordering. Moreover, we show that the development of the response with decreasing temperature in $\kappa$-NCS is steeper than that in $\kappa-\mathrm{Br}$. The results suggest that PIPS has a more significant effect on the structural ordering in $\kappa$-NCS than that in $\kappa-\mathrm{Br}$.

\section{EXPERIMENTAL}

Single crystals of the organic compounds were prepared electrochemically [14]. The crystals have layered structures, which consist of alternately stacked conducting layers of BEDT-TTF molecules and an insulating layer of $X$ along the $b$ axis for $\kappa-\mathrm{Br}$ and $\mathrm{Cl}$, and along the $a^{\star}$ axis for $\kappa$-NCS. The chemical substitution of $X$ corresponds to a change in unit-cell volumes in the same way as pressure. Figures 1(a) and 1(b) show a schematic of the BEDT-TTF molecular arrangement and the phase diagram [4]. The application of pressure increases the effective electron correlation $t / U$, where $t$ symbolizes the transfer integral between the dimers of BEDT-TTF molecules $t_{\mathrm{d}}$ and $t_{\mathrm{d}}^{\prime}$, and $U$ is the on-site Coulomb repulsion. The ground state is a superconductor in $\kappa$ - $\mathrm{Br}$ and $\kappa$-NCS and the Mott insulator in $\kappa-\mathrm{Cl}$. The glasslike transition is observed at $T_{\mathrm{g}} \sim 80 \mathrm{~K}$ for $\kappa-\mathrm{Br}$ and at $70 \mathrm{~K}$ for $\kappa-\mathrm{NCS}$ and $\kappa-\mathrm{Cl}$.

The optical measurements were performed using 120-fs pulses centered at $400 \mathrm{~nm}$ for a pump $\left(7-250 \mu \mathrm{J} / \mathrm{cm}^{2}\right)$ and $800 \mathrm{~nm}$ for a probe from a cavity-dumped Ti:sapphire oscillator with a repetition rate of $54 \mathrm{kHz}$ to avoid the heating effect. The pump and probe beams were coaxially overlapped by a dichroic mirror and irradiated perpendicular to the conducting plane. A polarization dependence of the probe is measured by rotating a half-wave plate, where $\theta$ is measured from the direction tilted by $45^{\circ}$ from the $b$ axis for $\kappa$-NCS. For $\kappa-\mathrm{Br}, c$ axis corresponded to the directions of $45^{\circ}$ and $225^{\circ}$, which was inferred from the previous report [15]. Optical penetration depth is roughly estimated as $13 \mu \mathrm{m}$ for pump and $3 \mu \mathrm{m}$ for probe in $\kappa$-NCS, respectively, which are almost identical to those in $\kappa-\mathrm{Br}$, meaning that bulk electronic properties are measurable. The samples were carefully cooled with a helium flow cryostat at a rate of $0.1-1.0 \mathrm{~K} / \mathrm{min}$ to avoid the disordering of the terminal ethylene groups of the BEDT-TTF molecules. 

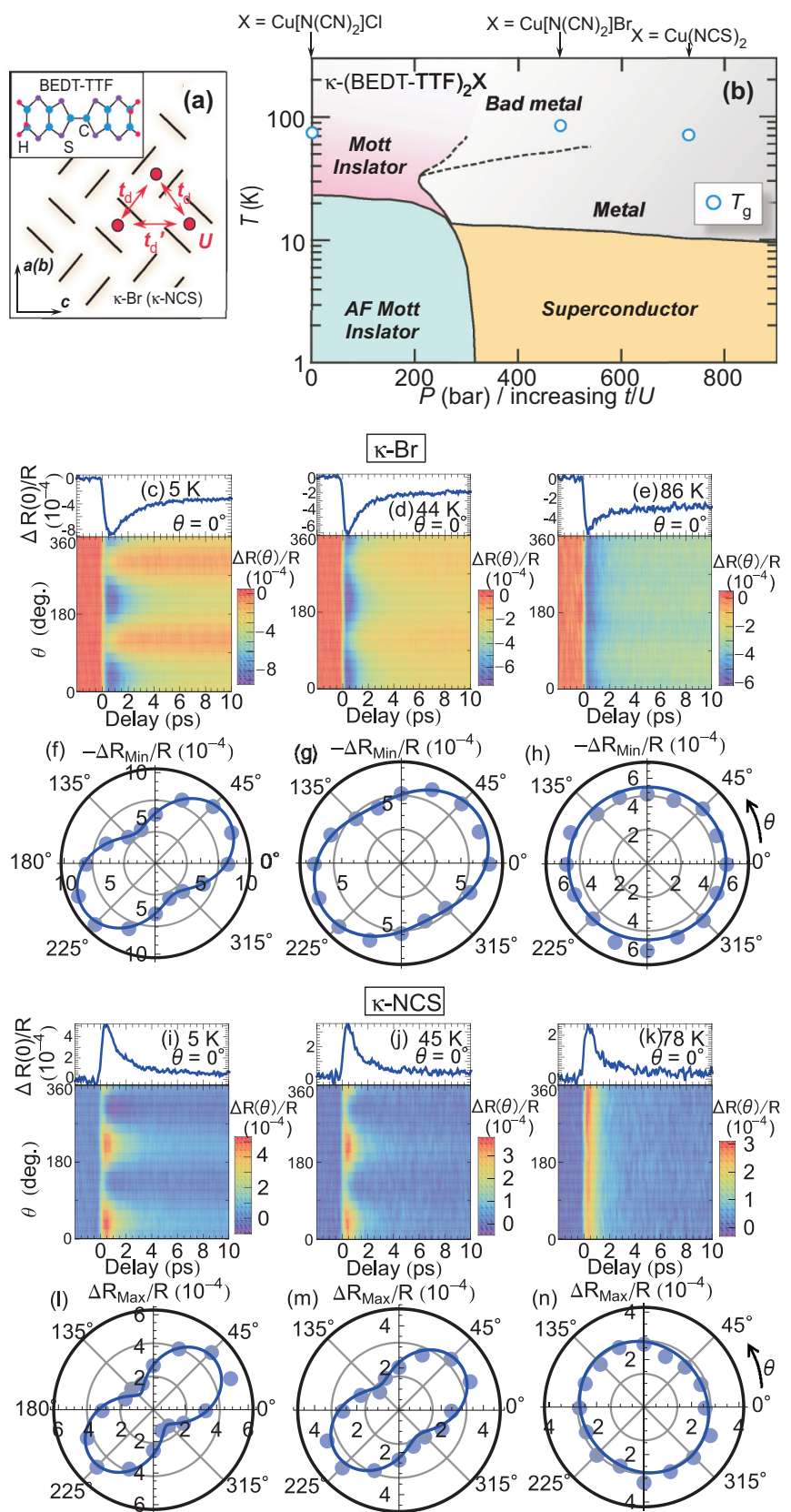

FIG. 1. (a) BEDT-TTF molecular arrangement of conducting layer. (b) Pressure-temperature phase diagram of $\kappa$-(BEDT-TTF) ${ }_{2} X$ system. (c)-(e) $\Delta R / R$ transients for $\theta=0^{\circ}$ and intensity plots of $\Delta R / R$ as a function of $\theta$ in $\kappa-\mathrm{Br}$ at $T=5,44$, and $86 \mathrm{~K}$, respectively. (f)-(h) Polar plots of the minimum values of $\Delta R / R$. (i)-(k) $\Delta R / R$ transients for $\theta=0^{\circ}$ and intensity plots of $\Delta R / R$ as a function of $\theta$ in $\kappa$-NCS at $T=5,45$, and $78 \mathrm{~K}$, respectively. (1)-(n) Polar plots of the maximum values of $\Delta R / R$. The solid lines are the results fitted by Eq. (1).

In the optical pump-probe experiments, the pump beam excites carriers to a nonequilibrium high-energy state. This results in immediate relaxation of the excited carriers to states near the Fermi level by electron-electron and electron-phonon scattering [16]. When a gap for electronic excitation, such as a superconducting gap or pseudogap, is present, a relaxation bottleneck may occur and the carriers accumulate just above the gap $[17,18]$. The probe beam delayed with the time measures the change of reflectivity $\Delta R$ which is connected with the nonequilibrium distribution of the carriers as a function of time.

\section{RESULTS}

Figures 1(c)-1(e) show transient reflectivity changes $\Delta R / R$ for $\theta=0^{\circ}$ and intensity plots for $63 \mu \mathrm{J} / \mathrm{cm}^{2}$ as a function of $\theta$ in $\kappa-\mathrm{Br}$ at $T=5,44$, and $86 \mathrm{~K}$, respectively. The polar plots of the signal amplitude of $\Delta R / R$ are shown in Figs. 1(f)-1(h). At $86 \mathrm{~K}, \Delta R / R$ showed no probe-polarization dependence. This has been attributed to the electron energy relaxation in the metallic state. At $44 \mathrm{~K}, \Delta R / R$ become $\theta$-dependent where the signal enhanced along the direction $45^{\circ}$ and $225^{\circ}$. The direction of anisotropy is unchanged down to $5 \mathrm{~K}\left(<T_{\mathrm{c}}\right)$. Figures $1(\mathrm{i})-1(\mathrm{n})$ show the probe angular dependencies of $\Delta R / R$ for $134 \mu \mathrm{J} / \mathrm{cm}^{2}$ at $T=5,45$, and $78 \mathrm{~K}$, respectively, in $\kappa$-NCS. As is the case in $\kappa-\mathrm{Br}, \Delta R / R$ is independent of $\theta$ at $78 \mathrm{~K}$, while, at 45 and $5 \mathrm{~K}$, the similar anisotropic responses for the probe polarization are observed. In the previous researches, the emergence of anisotropy arises from electronic or structural transitions accompanied by spontaneous symmetry breaking $[11,12]$. As will be mentioned later, the glasslike structural transition [8], which is not applicable to the concept of symmetry breaking, can play an important role for the development of the anisotropy in our measurements.

For further analysis, the data are decomposed into the anisotropic and isotropic components by fitting the following form:

$$
\frac{\Delta R(\theta)}{R} \propto \Delta R_{\mathrm{ani}} \cos \left(2 \theta-\phi_{\mathrm{c}}\right)+\Delta R_{\mathrm{iso}},
$$

to the angular dependence of $\Delta R(\theta) / R$, where $\Delta R_{\text {ani }}, \Delta R_{\text {iso }}$ and $\phi_{\mathrm{c}}$ denote anisotropic and isotropic components of $\Delta R / R$ and a phase, respectively. Figures 2(a) and 2(c), and 2(b) and 2(d) show the anisotropic transients $\Delta R_{\text {ani }} / R$ and the isotropic transient $\Delta R_{\text {iso }} / R$, respectively, for various temperatures in $\kappa-\mathrm{Br}$ and $\kappa$-NCS, respectively. The $\Delta R_{\text {ani }} / R$ is found to increase with decreasing temperature, whereas $\Delta R_{\text {iso }} / R$ seems to be unchanged. $\phi_{\mathrm{c}}$ has an almost constant value $\sim 45^{\circ}$ at low temperatures for both compounds. The $\Delta R_{\text {ani }} / R$ transients in $\kappa-\mathrm{Br}$ are quite similar to those in $\kappa$-NCS for various temperatures. This clearly indicates that the anisotropic response in each data arises from the same origin. Figures $2(\mathrm{e})$ and 2(f) show $\Delta R_{\text {ani }} / R$ at around $70 \mathrm{~K}$ in $\kappa$ - $\mathrm{Br}$ and $\kappa$-NCS, respectively. We found that, in $\kappa-\mathrm{Br}$, the signal gradually grows with decreasing temperature, while it abruptly develops below $70 \mathrm{~K}$ in $\kappa$-NCS.

The inset of Fig. 3(a) shows temperature dependence of signal amplitude for the isotropic component $A_{\text {iso }}$. The $A_{\text {iso }}$ values are almost constant in the whole temperature range, indicating that carrier relaxation of the metal state is dominant in the isotropic channel even at low temperatures. In contrast, the signal amplitude for the anisotropic component $A_{\text {ani }}$ is found to increase gradually from $\sim 80 \mathrm{~K}$, which is close to $T_{\mathrm{g}}$ with decreasing temperature as plotted in the left axis of Fig. 3(a). Since $\Delta R / R$ is related with the photoinduced carrier density, the change of $\Delta R / R$ indicates the development of 

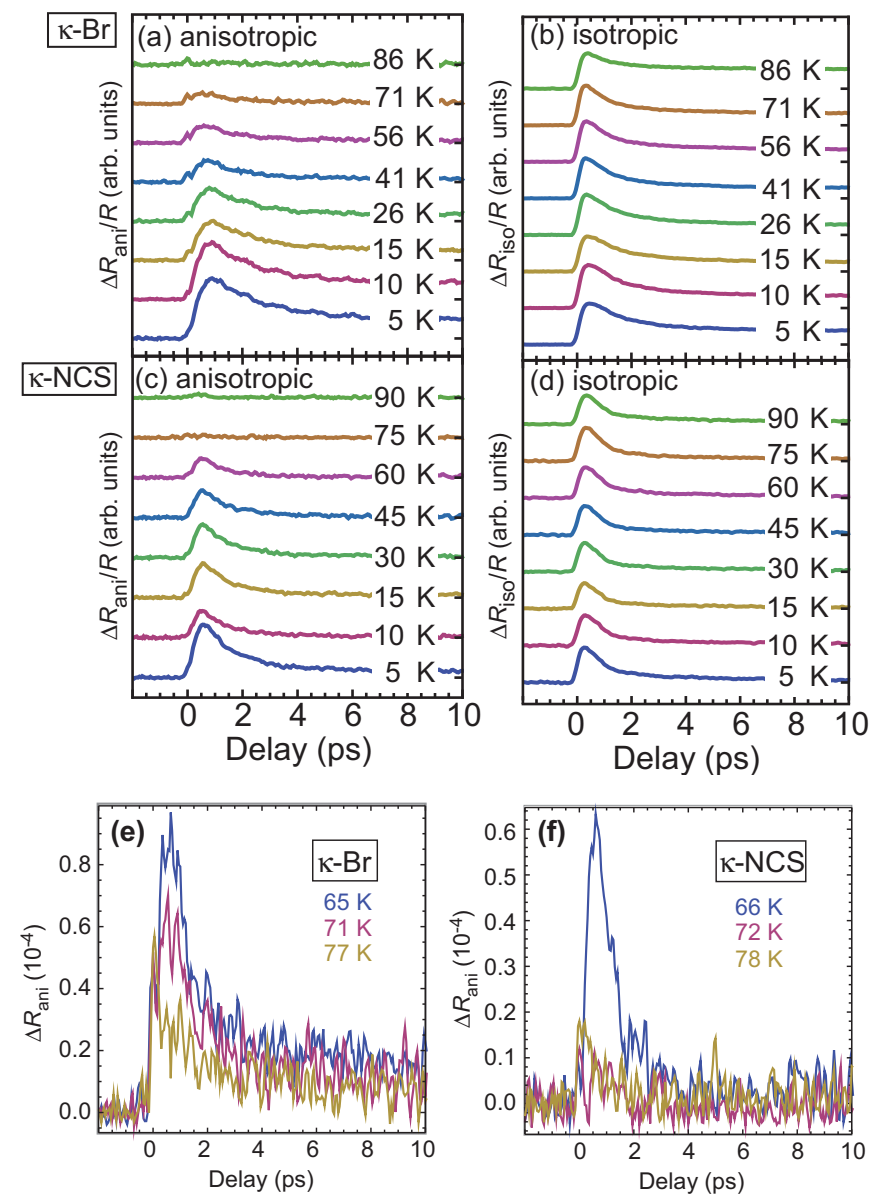

FIG. 2. (a),(b) $\Delta R / R_{\text {ani }}$ and $\Delta R_{\text {iso }} / R$ transients for various temperatures in $\kappa$ - $\mathrm{Br}$, respectively. (c),(d) $\Delta R / R_{\text {ani }}$ and $\Delta R_{\text {iso }} / R$ transients for various temperatures in $\kappa$-NCS, respectively. The data are shifted for clarity. (e),(f) $\Delta R / R_{\text {ani }}$ transients at around $70 \mathrm{~K}$ in $\kappa-\mathrm{Br}$ and $\kappa$-NCS, respectively.

energy gap. The similar gradual increase of $\Delta R / R$ has been observed as pseudogap (PG) responses in the superconducting cuprates [11]. In this case, the energy gap can be evaluated with the following form [18]:

$$
A(T) \propto\left[1+B T \exp \left(-\frac{\Delta_{\mathrm{PG}}}{T}\right)\right]^{-1},
$$

where $B$ and $\Delta_{\mathrm{PG}}$ are a variable constant for the fit and temperature-independent energy gap, respectively. We note that the model assumes the weak photoexcitation condition where fluence is below the saturation threshold value. As shown in Figs. 3(a) and 3(b), the data are fitted well by the model (the solid line) and yield $\Delta_{\mathrm{PG}} \approx 7 \mathrm{meV}$ for $\kappa$-Br and $\Delta_{\mathrm{PG}} \approx 46 \mathrm{meV}$ for $\kappa-\mathrm{NCS}$. Hereafter, the development of $A_{\text {ani }}$ observed above $T_{\mathrm{c}}$ is called as the $P G$ response.

In $\kappa-\mathrm{Br}$, the direction of anisotropy and its temperature dependence agree with those in a previous work [15]. In this study, the metallic-insulating PIPS has been suggested as a possible origin for the PG response. According to this scenario, the irradiation of pump pulse will lead to modulation of $t / U$ [15], which can act as a negative pressure on the electronic state. As a result, a part of the sample changes from

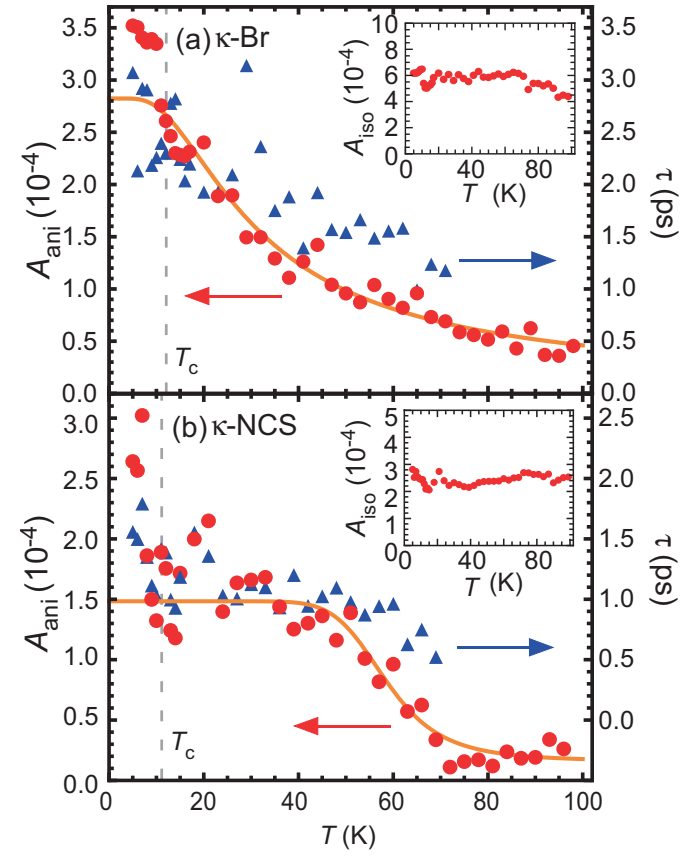

FIG. 3. (a) Temperature dependence of amplitude of anisotropic component $A_{\text {ani }}$ (left axis) and decay time (right axis) in $\kappa$-Br. The inset shows temperature dependence of amplitude of anisotropic component $A_{\text {iso }}$. (b) Temperature dependence of $A_{\text {ani }}$ (left axis) and decay time (right axis) in $\kappa$-NCS. The inset shows temperature dependence of $A_{\text {iso }}$. The solid lines display the results fitted by Eq. (2).

metal to insulator, leading to microscopic or mesoscopic phase separation in real space and/or a gap opens at different points in the momentum space. In the previous measurement, the signal amplitude of $\Delta R / R$ increases gradually with lowering temperature [15], which is quite similar to our results. The fit using Eq. (2) yields $\Delta_{\mathrm{PG}} \approx 16 \mathrm{meV}$, which is somewhat larger than ours. This difference may be due to the different excitation condition with the pump pulse wavelength of $1300 \mathrm{~nm}$ in the previous study.

The temperature dependence of $A_{\text {iso }}$ for $\kappa$-NCS is shown in the inset of Fig. 3(b). The $A_{\text {iso }}$ shows no considerable change for temperature variation. Strikingly, as shown in Fig. 3(b), the PG response emerges at $T_{\mathrm{g}} \sim 70 \mathrm{~K}$, which is quite similar to that in $\kappa$-Br, but has a more abrupt change than $\kappa$-Br. The $\Delta_{\mathrm{PG}}$ value is quite larger than that of $\kappa-\mathrm{Br}$.

The relaxation times $\tau$ are estimated by fitting the rapid decline of transient with a single exponential function, which are plotted as a function of temperature for $\kappa-\mathrm{Br}$ and $\kappa$-NCS in the right axis of Figs. 3(a) and 3(b), respectively. Although the $\tau$ value $\sim 1.5 \mathrm{ps}$ for $\kappa-\mathrm{Br}$ at $60 \mathrm{~K}$ is slightly larger than $\sim 1.0 \mathrm{ps}$ for $\kappa$-NCS, those temperature dependencies are very similar to each other, suggesting that the PG responses arise from the PIPS in $\kappa$-NCS as well as in $\kappa$-Br.

For both compounds, the optical conductivity measurements have suggested that any type of gap is not developed above $T_{\mathrm{c}}$ [19-21]. Moreover, an anomaly which is due to some ordered states, such as charge density wave (CDW), has not been reported in other measurements. The absence of the gapped states above $T_{\mathrm{c}}$ strongly indicate that the PG response is originated from the photoinduced states. 

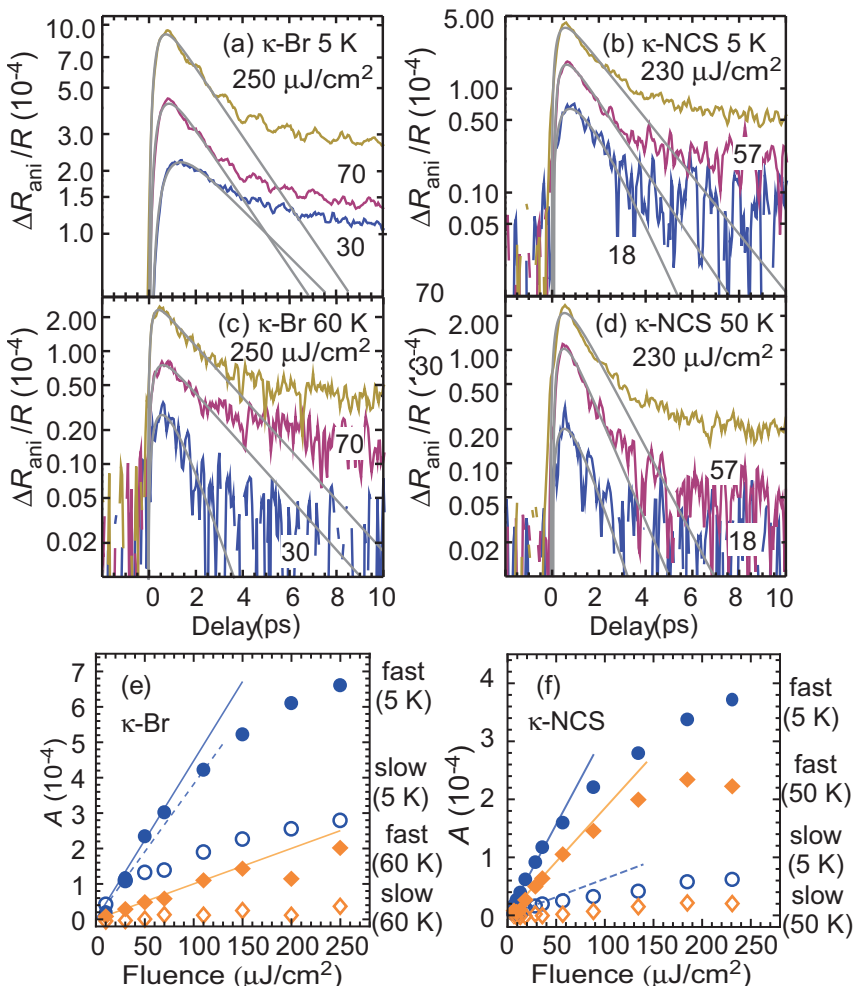

FIG. 4. (a),(b) $\Delta R / R$ transients of the anisotropic components for various pump fluences at $5 \mathrm{~K}$ in $\kappa$ - $\mathrm{Br}$ and $\kappa$-NCS, respectively. (c),(d) $\Delta R / R$ transients of the anisotropic components for various pump fluences at $60 \mathrm{~K}$ in $\kappa-\mathrm{Br}$ and at $50 \mathrm{~K}$ in $\kappa$-NCS, respectively. (e),(f) Fluence dependence of $B_{\mathrm{f}}$ and $B_{\mathrm{f}}$ which correspond to the fast and slow decay components for $\kappa$-Br and $\kappa$-NCS, respectively. The solid and dashed lines denote the linear fluence dependence.

In Fig. 3 , the $A_{\text {ani }}$ slightly jumps just at $T_{\mathrm{c}}$, deviating from the model in both compounds. These are consistent with the formation of SC gap, indicating that two relaxation components associated with $\mathrm{PG}$ and $\mathrm{SC}$ gap are included below $T_{\mathrm{c}}$ in the anisotropic channel. To verify the SC contribution below $T_{\mathrm{c}}$, we show the results of anisotropic component $\Delta R_{\text {ani }} / R$ on a logarithmic scale at various pump fluences in Figs. 4(a)-4(d). In both compounds, the $\Delta R_{\text {ani }} / R$ increases as pump fluence increases and shows a long-lived component at $5 \mathrm{~K}$, which shows a deviation from the single exponential function (the solid lines) at any fluences [Figs. 4(a) and 4(b)]. In contrast, at temperatures above $T_{\mathrm{c}}$ [Figs. 4(c) and 4(d)], the long-lived component is almost absent except for the saturation condition, where the temperature-independent contribution cannot be removed for high fluence excitation. These results indicate that the substantial increase of the $A_{\text {ani }}$ below $T_{\mathrm{c}}$ comes from the long-lived component.

By assuming an infinite decay time of the long-lived component, we fitted the data with the form $B_{\mathrm{f}} \exp \left(-t / \tau_{\mathrm{f}}\right)+$ $B_{1}$, where $B_{\mathrm{f}}$ and $B_{1}$ are amplitudes of the fast decay and long-lived components, respectively, and $\tau_{\mathrm{f}}$ is the decay time of the fast component. Figures 4(e) and 4(f) represent the fluence dependencies of the $B_{\mathrm{f}}$ and $B_{1}$ in $\kappa$ - $\mathrm{Br}$ and $\kappa$-NCS, respectively. Both $B_{\mathrm{f}}$ and $B_{1}$ increase linearly (solid and dashed lines) with increasing fluence at low fluences and saturate at high fluences below $T_{\mathrm{c}}$. The saturation behavior corresponds to the fact that
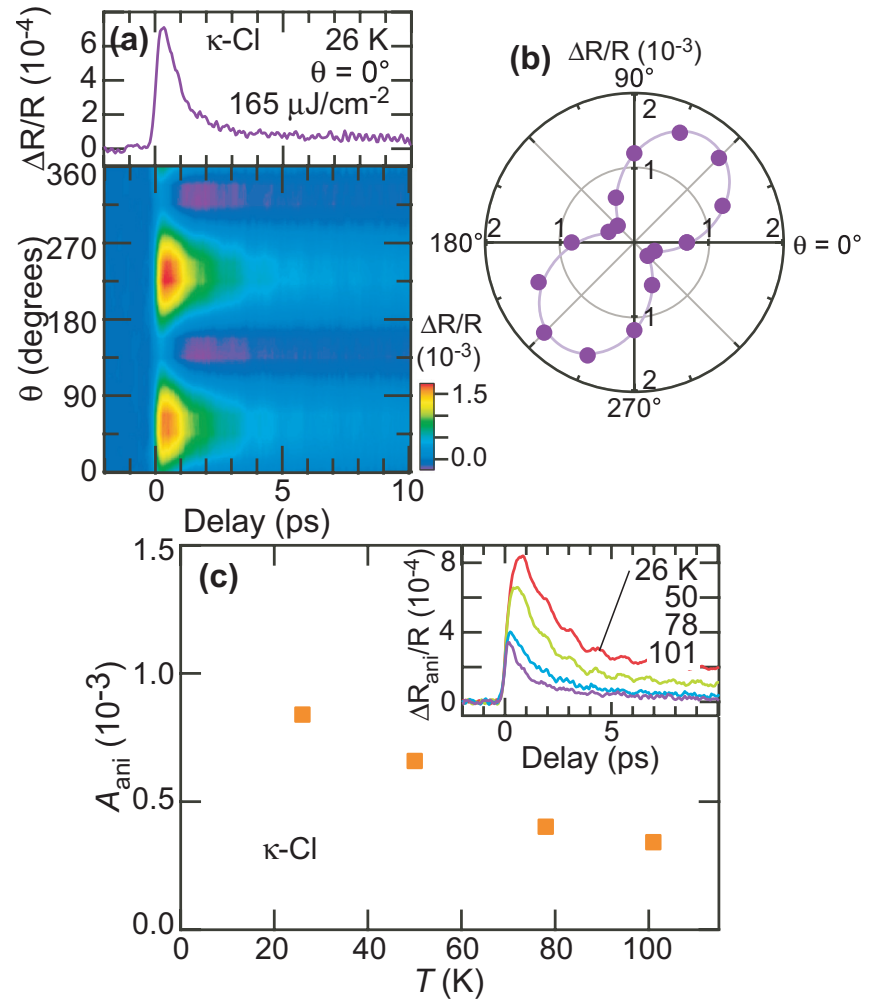

FIG. 5. (a) $\Delta R / R$ transients for $\theta=0^{\circ}$ and intensity plots of $\Delta R / R$ as a function of $\theta$ at $20 \mathrm{~K}$ in $\kappa-\mathrm{Cl}$. (b) Polar plots of the maximum values of $\Delta R / R$. The solid lines are the results fitted by Eq. (1). (c) Temperature dependence of amplitude of anisotropic component $A_{\text {ani }}$. The inset shows $\Delta R / R$ transients of the anisotropic components for typical temperatures.

the excited states are limited, such as the photoinduced collapse of the energy gap. Above $T_{\mathrm{c}}$, the $B_{\mathrm{f}}$ again shows saturation but the $B_{1}$ is negligibly small. Consequently, we can conclude that the fast decay and long-lived components are ascribed to the carrier relaxation of PG and SC states, respectively, both of which coexist below $T_{\mathrm{c}}$.

The coexistence between SC and PG responses below $T_{\mathrm{c}}$ is compatible with the PIPS. Above $T_{\mathrm{c}}$, both compounds belong to the metallic phase under equilibrium condition. When the sample is irradiated by the pump pulse, the insulating (gapped) regions are induced in the real and/or momentum spaces. The remaining metallic regions (Fermi surface) can contribute the SC condensate. Thus, below $T_{\mathrm{c}}$, superconductor-insulator phase separation will be realized. Because the relaxation time of the SC response is longer than that of the PG response, the phase separation will be eliminated with time faster than recovery of the SC ordering.

\section{DISCUSSION}

In what follows, a relationship between the anisotropy of the PG response and PIPS is discussed. The emergence of anisotropy has been discussed only in electronic or structural ordering systems, which is characterized by spontaneous symmetry breaking $[11,12]$. For example, in the case of two-dimensional charge density wave (CDW) [12], the electronic system has an orientation along a CDW wave vector 
below the transition temperature, leading to rotational as well as translational symmetry breaking. In the pump-probe measurements, the orientation is destroyed transiently by the irradiation of pump pulse. By probing the relaxation process with different probe polarizations, a recovery of the orientation can be detected as a transient polarization anisotropy. However, the PIPS itself cannot imply any symmetry breaking because the insulating phase can be caused by the photoinduced modulation of $t / U$, corresponding to the Mott state. To confirm this, we show the results of $\kappa-\mathrm{Cl}$, where the ground state is the Mott insulator. Figures 5(a) and 5(b) show $\Delta R / R$ as a function of $\theta$ in $\kappa-\mathrm{Cl}$ at $T=26 \mathrm{~K}$ and the polar plots of the signal amplitude of $\Delta R / R$, respectively. The anisotropic response for the probe polarization was again observed. The inset of Fig. 5(c) shows the anisotropic component of $\Delta R / R$ after decomposition using Eq. (1) for typical temperatures. As plotted in Fig. 5(c), the signal amplitude gradually increases with decreasing temperature. This behavior, which is in good agreement with the previous result [15], is explained by the presence of the Mott gap. Because the Mott state is realized without symmetry breaking, the results indicate that the anisotropy does not arise from symmetry breaking of the electronic system.

Instead, the glasslike structural transition is responsible for the anisotropy where $T_{\mathrm{g}} \sim 70 \mathrm{~K}$ in $\kappa-\mathrm{Cl}[8]$ is in good agreement with the temperature at which the Mott response is visible. The thermal-expansion measurements show the occurrence of some short-range ordering, which can be associated with the internal degrees of freedom in molecules, such as the terminal ethylene groups of BEDT-TTF molecules [8] or the anion molecules $X$ [9]. Although the glasslike transition is not characterized by spontaneously symmetry breaking [8], such local molecular ordering is expected to provide an orientation below $T_{\mathrm{g}}$ in a similar way to the case of symmetry breaking systems. Such fine structural orientation cannot be detected by the x-ray-diffraction measurements because the experimental resolution is not sufficient [9]. On the other hand, our pump-probe measurements can probe it effectively through photoinduced dynamics with recovery of the structural ordering as an increase of anisotropy. However, we note that the glasslike transition itself cannot be accompanied by an opening of the energy gap [19-21], although it can influence the electronic states [7]. Therefore, the results from $\kappa-\mathrm{Cl}$ indicate that the photoexcitation does not only induce carriers but also destroys the structural ordering transiently, enhancing the anisotropic response for the probe.

The foregoing discussion allows us to conclude that the glasslike structural transition plays a vital role for the PIPS in $\kappa-\mathrm{Br}$ and $\kappa$-NCS, where the $T_{\mathrm{g}}$ values are $\sim 80$ and $70 \mathrm{~K}$, respectively. The Mott insulating state is partially induced by the photoinduced change in the molecular configuration, which corresponds to the modulation of $t / U$. Simultaneously, in the photoinduced Mott region, the local structural ordering should be destroyed in the same way as $\kappa-\mathrm{Cl}$. As a result, the carrier relaxation with recovery of the structural ordering is observed as the anisotropic PIPS response.

We now consider the qualitative difference in the temperature dependencies between $\kappa-\mathrm{Br}$ and $\kappa$-NCS. In $\kappa-\mathrm{Br}$ [Fig. 3(a)], the signal is gradually increased as the temperature is decreased, whereas in $\kappa$-NCS, a steep increase is observed with decreasing temperature as compared to $\kappa$ - $\mathrm{Br}$ [Fig. 3(b)]. The results indicate that the anisotropy is more enhanced in $\kappa$ NCS than in $\kappa$-Br. In the process of PIPS, electronic excitation by the pump pulse can induce not only the modulation of $t / U$ but also the structural disordering of molecules. Because the electronic state of $\kappa$-NCS is located far from the Mott phase boundary as compared to that of $\kappa-\mathrm{Br}$, the modulation of $t / U$ in $\kappa$-NCS is more efficient than that in $\kappa$-Br. In a similar way, a more significant disordering is expected to be induced more in $\kappa$-NCS than in $\kappa$-Br. This may explain why the PIPS response varies steeply below $T_{\mathrm{g}}$ in $\kappa$-NCS.

\section{CONCLUSIONS}

To summarize, by performing polarized pump-probe spectroscopy, we have shown that the PIPS is closely related to the local structural ordering in $\kappa-\mathrm{Br}$ and $\kappa$-NCS. The steep variation in temperature dependence of $\kappa$-NCS indicates that the disordering of molecules is more significantly induced by photoexcitation in $\kappa$-NCS than in $\kappa-\mathrm{Br}$.

\section{ACKNOWLEDGMENTS}

We would like to thank T. Mertelj, D. Mihailovic, and K. Ichimura for valuable discussions. This work was supported by JSPS KAKENHI Grant No. 15K17685.
[1] A. Ardavan, S. Brown, S. Kagoshima, K. Kanoda, K. Kuroki, H. Mori, M. Ogata, S. Uji, and J. Wosnitza, J. Phys. Soc. Jpn. 81, 011004 (2012).

[2] Y. Tokura, J. Phys. Soc. Jpn. 75, 011001 (2006).

[3] P. Garoche, R. Brusetti, and K. Bechgaard, Phys. Rev. Lett. 49, 1346 (1982).

[4] B. J. Powell and R. H. McKenzie, J. Phys.: Condens. Matter 18, R827 (2006).

[5] R. H. McKenzie, Science 278, 820 (1997).

[6] M. A. Tanatar, T. Ishiguro, T. Kondo, and G. Saito, Phys. Rev. B 59, 3841 (1999).

[7] H. Akutsu, K. Saito, and M. Sorai, Phys. Rev. B 61, 4346 (2000).

[8] J. Muller, M. Lang, F. Steglich, J. A. Schlueter, A. M. Kini, and T. Sasaki, Phys. Rev. B 65, 144521 (2002).
[9] A. U. B. Wolter, R. Feyerherm, E. Dudzik, S. Sullow, Ch. Strack, M. Lang, and D. Schweitzer, Phys. Rev. B 75, 104512 (2007).

[10] O. J. Taylor, A. Carrington, and J. A. Schlueter, Phys. Rev. B 77, 060503(R) (2008).

[11] Y. Toda, F. Kawanokami, T. Kurosawa, M. Oda, I. Madan, T. Mertelj, V. V. Kabanov, and D. Mihailovic, Phys. Rev. B 90, 094513 (2014).

[12] S. Tsuchiya, Y. Sugawara, S. Tanda, and Y. Toda, J. Opt. 17, 085501 (2015).

[13] K. Nakagawa, S. Tsuchiya, J. Yamada, and Y. Toda, J. Supercond. Nov. Magn. 29, 3065 (2016).

[14] H. Anzai, J. M. Delrieu, S. Takasaki, S. Nakajima, and J. Yamada, J. Cryst. Growth 154, 145 (1995). 
[15] Y. Toda, T. Mertelj, T. Naito, and D. Mihailovic, Phys. Rev. Lett. 107, 227002 (2011).

[16] P. B. Allen, Phys. Rev. Lett. 59, 1460 (1987).

[17] A. Rothwarf and B. N. Taylor, Phys. Rev. Lett. 19, 27 (1967).

[18] V. V. Kabanov, J. Demsar, B. Podobnik, and D. Mihailovic, Phys. Rev. B 59, 1497 (1999).
[19] D. Faltermeier, J. Barz, M. Dumm, M. Dressel, N. Drichko, B. Petrov, V. Semkin, R. Vlasova, C. Meziere, and P. Batail, Phys. Rev. B 76, 165113 (2007).

[20] M. Dumm, D. Faltermeier, N. Drichko, M. Dressel, C. Meziere, and P. Batail, Phys. Rev. B 79, 195106 (2009).

[21] A. Ugawa, G. Ojima, K. Yakushi, and H. Kuroda, Phys. Rev. B 38, 5122(R) (1988). 